\title{
Tubular injury triggers podocyte dysfunction by $\beta$-catenin-driven release of MMP-7
}

\author{
Roderick J. Tan, ${ }^{1}$ Yingjian Li, ${ }^{2}$ Brittney M. Rush, ${ }^{1}$ Débora Malta Cerqueira, ${ }^{3}$ Dong Zhou, ${ }^{2}$ Haiyan Fu, ${ }^{4}$ \\ Jacqueline Ho, ${ }^{3}$ Donna Beer Stolz, ${ }^{5}$ and Youhua Liu' ${ }^{2,4}$ \\ ${ }^{1}$ Renal-Electrolyte Division, Department of Medicine, ${ }^{2}$ Department of Pathology, and ${ }^{3}$ Division of Pediatric Nephrology, \\ Department of Pediatrics, University of Pittsburgh School of Medicine, Pittsburgh, Pennsylvania, USA. ${ }^{4}$ Division of \\ Nephrology, Nanfang Hospital, Southern Medical University, Guangzhou, China. ${ }^{5}$ Department of Cell Biology, University of \\ Pittsburgh School of Medicine, Pittsburgh, Pennsylvania, USA.
}

Proteinuric chronic kidney disease (CKD) remains a major health problem worldwide. While it is well established that the progression of primary glomerular disease induces tubulointerstitial lesions, how tubular injury triggers glomerular damage is poorly understood. We hypothesized that injured tubules secrete mediators that adversely affect glomerular health. To test this, we used conditional knockout mice with tubule-specific ablation of $\beta$-catenin (Ksp- $\beta$-cat ${ }^{-/-}$) and subjected them to chronic angiotensin II (Ang II) infusion or Adriamycin. Compared with control mice, Ksp- $\beta$-cat ${ }^{-/-}$ mice were dramatically protected from proteinuria and glomerular damage. MMP-7, a downstream target of $\beta$-catenin, was upregulated in treated control mice, but this induction was blunted in the Ksp- $\beta$-cat ${ }^{-1-}$ littermates. Incubation of isolated glomeruli with MMP-7 ex vivo led to nephrin depletion and impaired glomerular permeability. Furthermore, MMP-7 specifically and directly degraded nephrin in cultured glomeruli or cell-free systems, and this effect was dependent on its proteolytic activity. In vivo, expression or infusion of exogenous MMP-7 caused proteinuria, and genetic ablation of MMP-7 protected mice from Ang II-induced proteinuria and glomerular injury. Collectively, these results demonstrate that $\beta$-catenin-driven MMP-7 release from renal tubules promotes glomerular injury via direct degradation of the key slit diaphragm protein nephrin.

Conflict of interest: The authors have declared that no conflict of interest exists.

Copyright: () 2019, American Society for Clinical Investigation.

Submitted: May 25, 2018

Accepted: November 14, 2019

Published: December 19, 2019

Reference information: /CI Insight. 2019;4(24):e122399. https://doi.org/10.1172/jici. insight.122399.

\section{Introduction}

Chronic kidney disease (CKD), or the permanent loss of renal function, is a growing health concern. In the United States alone CKD affects more than 35 million individuals (1). In particular, CKD characterized by abnormal urinary protein excretion (proteinuria) carries a high risk of progression to end-stage renal disease (2). Unfortunately, treatment for proteinuric CKD remains extremely limited. In this context, a better understanding of the underlying pathologic mechanisms is essential for developing more effective therapies for CKD.

Regardless of the initial etiology, $\mathrm{CKD}$ progression to end-stage renal disease is characterized by increasingly widespread lesions in different compartments of kidney parenchyma. While diseases that primarily affect the glomerular compartment of the nephron (e.g., glomerulonephritis) lead eventually lead to tubular injury and interstitial fibrosis (3), whether and how tubular injury affects the glomerular compartment are less well established and poorly understood. Studies show that kidney tubules can affect glomerular biology via signals originating from the macula densa, a specialized portion of the thick ascending limb of the loop of Henle (4). Considering the close apposition of renal tubules to glomeruli, it is possible that tubules influence glomerular health in other ways. Interestingly, in a model of acute kidney injury that specifically targeted renal tubules, Grgic et al. found that approximately $20 \%$ of the glomeruli developed sclerotic lesions (5). This was accompanied by an increase in proteinuria and suggested a significant tubular effect on glomerular health. However, the underlying mechanism by which tubular injury causes glomerular damage remains elusive.

$\mathrm{Wnt} / \beta$-catenin is a key signaling cascade that is critical for normal organogenesis. In this pathway, extracellular Wnts bind to cell-surface Frizzled receptors, transducing a signal that allows for the accumulation of cytoplasmic $\beta$-catenin, which then translocates to the nucleus and regulates target genes (6). 
While highly active during development, Wnt/ $\beta$-catenin signaling becomes quiescent in adult kidneys and reactivates during kidney injury (7-10). Activation of this signaling leads to a fibrotic response and promotes the progression of CKD via the upregulation of profibrotic mediators, such as fibronectin, the renin-angiotensin system, plasminogen activator inhibitor- 1 , and MMP-7 $(6,11-13)$. Induction of MMP-7 by Wnt/ $\beta$-catenin is particularly interesting, because it is the most robust $\beta$-catenin downstream target, and its expression primarily occurs in renal tubules (14-16). As a secreted protein that can be detected in the urine, MMP-7 is an effective noninvasive urinary biomarker for activation of $\mathrm{Wnt} / \beta$-catenin after kidney injury $(16,17)$.

In the current study, we evaluated kidney injury after chronic infusion of angiotensin II (Ang II) using conditional knockout mice with tubule-specific ablation of $\beta$-catenin (18). Interestingly, although the genetic mutation was restricted to renal tubules, we found that these mice had significant protection against glomerular injury and proteinuria, accompanied by a reduced MMP-7 expression. These results were replicated in an Adriamycin-induced proteinuria model. We further demonstrate that MMP-7 could degrade the slit diaphragm protein nephrin and impaired integrity of the glomerular filtration barrier. In vivo exposure of mice to elevated MMP-7 levels was sufficient to cause proteinuria, and global ablation of MMP-7 protected mice from Ang II-induced glomerular injury. Our findings illustrate that tubular injury promotes glomerular damage by $\beta$-catenin-driven release of MMP-7.

\section{Results}

Mice with tubule-specific ablation of $\beta$-catenin are protected from Ang II-induced albuminuria and glomerular injury. Tubule-specific $\beta$-catenin-knockout mice $\left(\mathrm{Ksp}-\beta\right.$-cat $\left.{ }^{-/}\right)$, in which the $\beta$-catenin gene was specifically disrupted in renal tubular epithelia by mating the $\beta$-catenin-floxed mice and Ksp-cadherin promoter-driven Cre recombinase-transgenic mice, were described and characterized previously (18). We exposed these mice to $1.5 \mathrm{mg} / \mathrm{kg} / \mathrm{d}$ Ang II via osmotic minipumps, which causes significant glomerular injury and albuminuria (19-22). In contrast to age- and sex-matched, $\beta$-catenin-floxed littermates (Ksp- $\beta$-cat $\left.{ }^{+/+}\right)$, Ksp- $\beta$-cat ${ }^{-/-}$mice were significantly protected from albuminuria at 14,21 , and 28 days after chronic Ang II infusion (Figure 1A). Gel electrophoresis of mouse urine samples confirmed that the majority of the protein in the urine was in fact albumin (Figure 1B). Immunohistochemical staining for megalin and cubilin did not reveal any qualitative differences between Ang II-treated Ksp$\beta$-cat ${ }^{+/+}$and $\mathrm{Ksp}-\beta$-cat ${ }^{-/-}$mice (Figure $1 \mathrm{C}$ ), suggesting it was unlikely that a defect in tubular reabsorption of albumin was the cause for the increase in urinary albumin excretion.

We then investigated differences in glomerular injury between $\mathrm{Ksp}-\beta$-cat ${ }^{+/+}$and $\mathrm{Ksp}-\beta-\mathrm{cat}^{-/-}$mice. At baseline there were no histologic differences between kidneys of naive Ksp- $\beta$-cat ${ }^{+/+}$mice and Ksp- $\beta$ $\mathrm{cat}^{-/-}$littermates (data not shown). However, kidneys from Ang II-treated $\mathrm{Ksp}-\beta-\mathrm{cat}^{+/+}$mice demonstrated increased glomerular injury, with focal areas of collapse and substantial proteinuria in Bowman's space. In contrast, treated Ksp- $\beta$-cat ${ }^{-1-}$ mice were protected from injury (Figure 1D). We further assessed the expression of nephrin and Wilms tumor 1 (WT1). Nephrin is a key component of the glomerular slit diaphragm and plays an important role in preventing urinary albumin excretion (23). WT1 is a transcription factor important for maintenance of normal podocyte differentiation (24). Control glomeruli at basal conditions demonstrated uninterrupted linear nephrin staining and abundant $\mathrm{WT}^{+}$nuclei. Significant disruptions in nephrin distribution and fewer $\mathrm{WT}^{+}$nuclei were observed in Ang II-treated Ksp- $\beta$-cat ${ }^{+/+}$ mice. The Ksp- $\beta-\mathrm{cat}^{-/-}$mice were partially protected from these Ang II effects (Figure 1, D-F). Immunoblotting analyses of kidney lysates confirmed the reduction of WT1 in Ksp- $\beta$-cat ${ }^{+/+}$mice compared with Ksp- $\beta$-cat ${ }^{-1-}$ littermates (Figure $1 G$ ).

Glomerular changes were also demonstrated on an ultrastructural level. Using transmission electron microscopy (TEM), we noted increased podocyte foot process effacement in the Ksp- $\beta$-cat ${ }^{+/+}$mice compared with untreated controls (Figure 2, A and B). The Ksp- $\beta$-cat ${ }^{-/-}$mice were largely protected from foot process effacement (Figure 2C). Similar changes were noted on scanning electron microscopy (SEM) (Figure 2, D-F). These data demonstrate that $\mathrm{Ksp}-\beta-\mathrm{cat}^{-1-}$ mice are protected from podocyte injury, in spite of the fact that the genetic deletion is limited to the renal tubules.

Tubule-specific ablation of $\beta$-catenin protects mice from renal fibrosis induced by Ang II infusion. We compared the late fibrotic response to Ang II between Ksp- $\beta$-cat ${ }^{+/+}$and Ksp- $\beta$-cat ${ }^{-/-}$mice. We found that Ksp- $\beta$-cat ${ }^{+/+}$ mice possessed more fibrosis than Ksp- $\beta-\mathrm{cat}^{-/-}$mice at time of sacrifice (Figure $3 \mathrm{~A}$ ). Fibronectin protein levels tended to be higher in the Ksp- $\beta$-cat ${ }^{+/+}$mice as well (Figure 3, B and C). Finally, serum creatinine levels were only significantly increased when comparing Ang II-treated Ksp- $\beta$-cat ${ }^{+/+}$mice with untreated controls. 


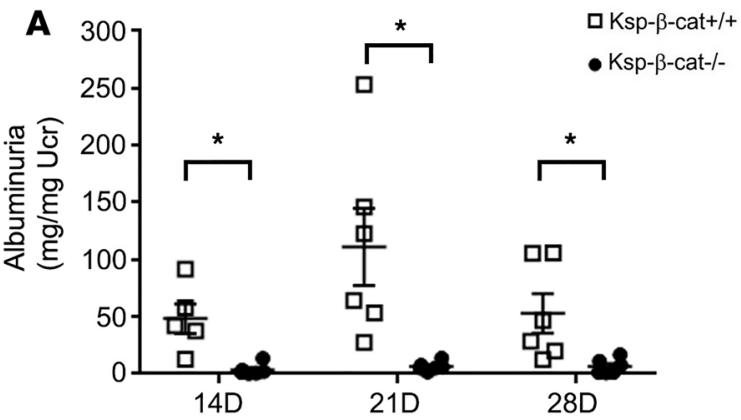

B

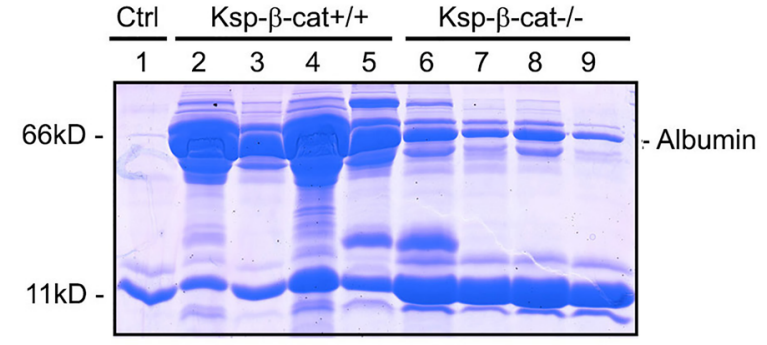

C

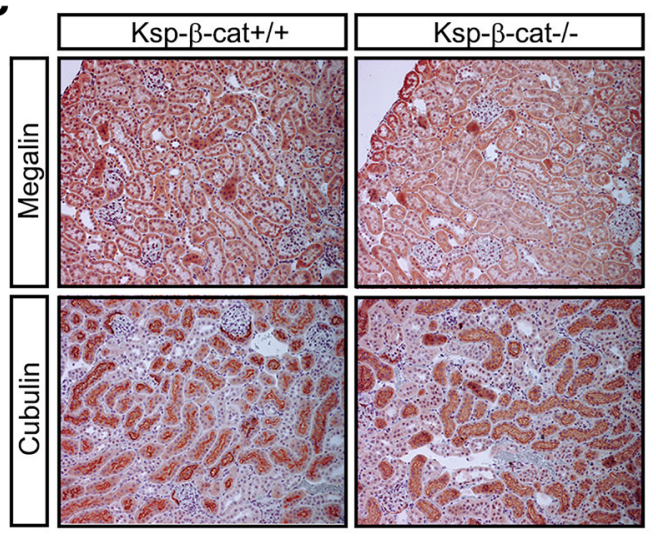

D
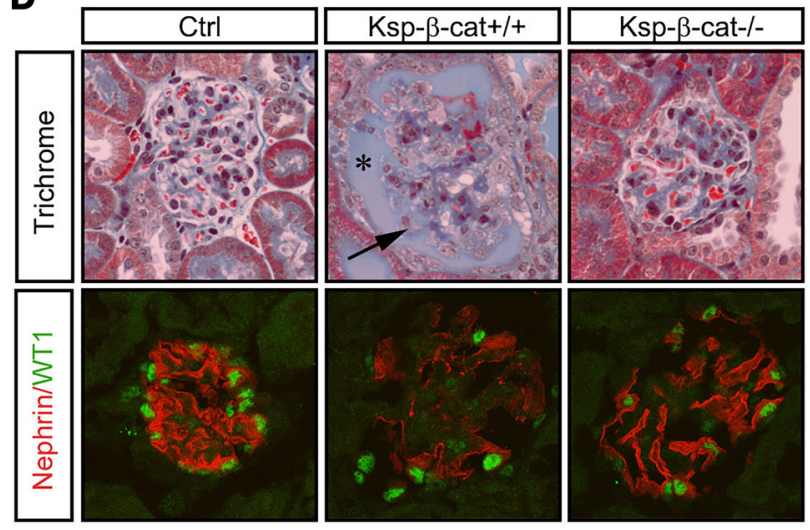

G
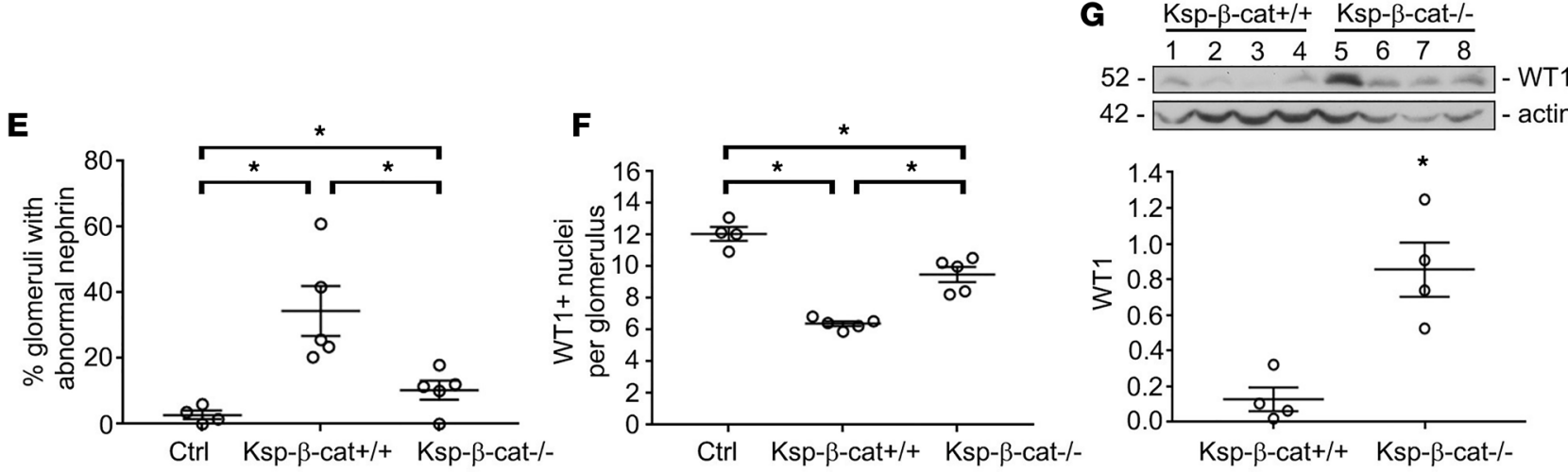

Figure 1. Tubule-specific $\beta$-catenin-knockout mice are protected from glomerular injury. The $\beta$-catenin-floxed mice $\left(\mathrm{Ksp}^{-} \beta\right.$-cat $\left.{ }^{+/+}\right)$and tubule-specific $\beta$-catenin-knockout (Ksp- $\beta$-cat ${ }^{-/-}$) mice were subjected to continuous angiotensin II (Ang II) infusions (1.5 mg/kg/d, osmotic minipump). (A) Urinary albumin

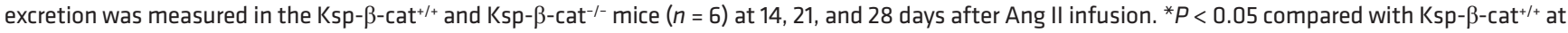
same time point, 1-way ANOVA. (B) Gel electrophoresis of urine samples shows the composition of the protein excreted in the urine. Albumin is indicated. Urine from an untreated control mouse (Ctrl, lane 1) was included for reference. (C) Levels of megalin and cubilin were not different between Ksp- $\beta$-cat ${ }^{+/+}$and $\mathrm{Ksp}-\beta$-cat ${ }^{-/-}$mice after Ang II infusion (original magnification, $\times 20$ ). (D) Evaluation of glomerular histology reveals increased glomerular damage in Ksp- $\beta$-cat ${ }^{+/+}$ compared with Ksp- $\beta$-cat ${ }^{-1-}$ mice. Note the high levels of protein in Bowman's space in the Ksp- $\beta$-cat ${ }^{+/+}$mice (asterisk), accompanied by marked glomerulosclerosis (arrow). In the Ksp- $\beta$-cat ${ }^{-/-}$mice, overall glomerular injury, including glomerulosclerosis, was minimal. A control (Ctrl, untreated mouse) glomerulus is provided for reference. Immunofluorescence revealed substantial disruption in nephrin and fewer WT1-positive podocytes in the Ksp- $\beta$-cat ${ }^{+/+}$compared Ksp- $\beta-$ cat $^{-/-}$mice (original magnification, $\times 40$ ). (E) Quantitation of the number of glomeruli with abnormal nephrin staining and (F) WT1-positive nuclei per glomeruli $\left(n=5,{ }^{*} P<0.05\right.$, 1-way ANOVA). (C) Immunoblot for WT1 showing depleted levels in the Ksp- $\beta$-cat ${ }^{+/+}$mice, compared with $\mathrm{Ksp}^{-\beta} \beta-\mathrm{cat}{ }^{-/-}$mice. ${ }^{*} P<0.05, t$ test.

The Ksp- $\beta$-cat ${ }^{-1}$ mice creatinine values were not significantly different from either of the other groups (Figure 3D). This indicates that not only are Ksp- $\beta$-cat ${ }^{-/-}$mice protected from glomerular injury, they are protected from chronic interstitial fibrosis and loss of renal function at late stages of disease.

Tubule-specific ablation of $\beta$-catenin inhibits renal MMP-7 expression. To determine the mechanism for why Ksp- $\beta$-cat ${ }^{-/-}$mice suffer less podocyte injury after Ang II exposure, we examined the levels of MMP-7, a secreted, low-molecular-weight metalloprotease that is a known direct target of $\beta$-catenin signaling (14). MMP-7 is not detectable by immunoblotting in normal kidneys (14). After Ang II treatment, MMP-7 levels 

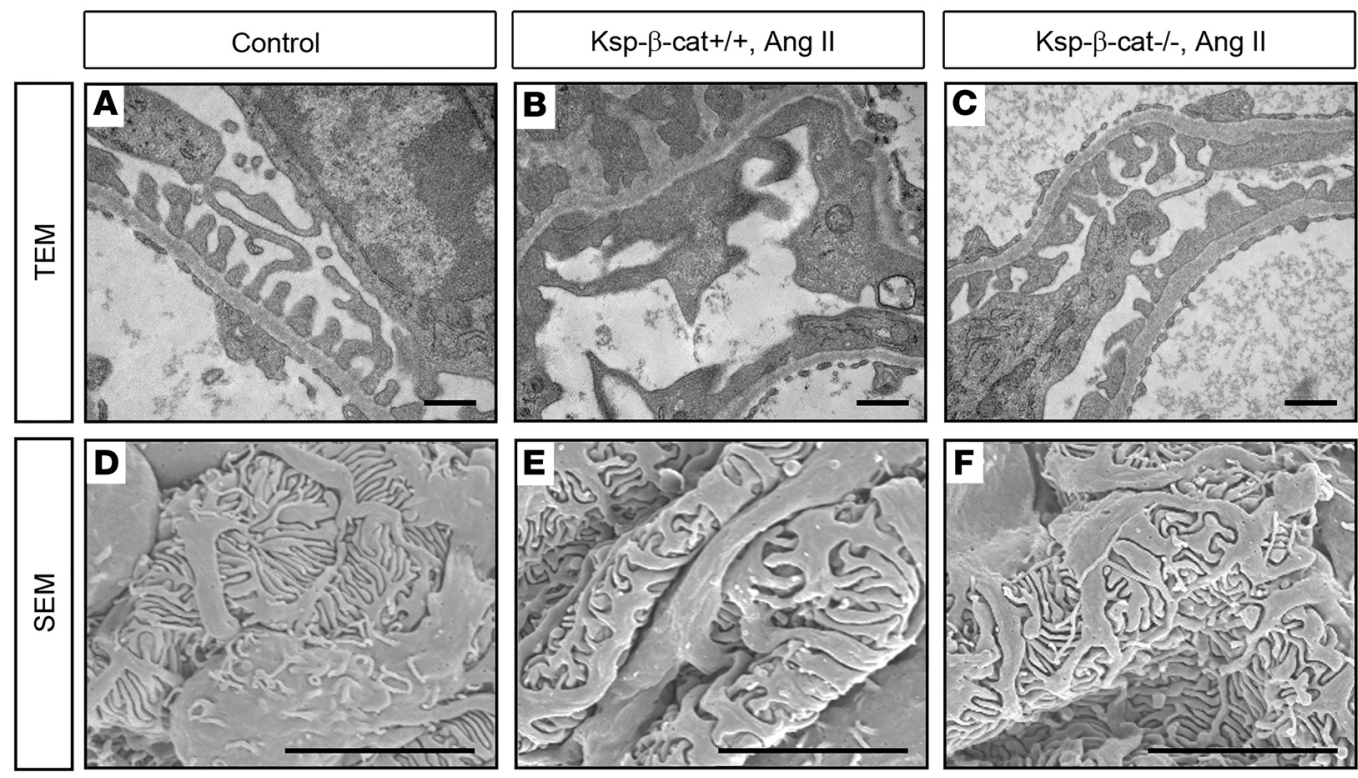

Figure 2. Podocyte foot process integrity is preserved in Ksp- $\beta$-cat ${ }^{-/-}$mice after Ang II infusion. Mice were treated as in Figure 1. (A-C) Transmission electron microscopy (TEM) showing extensive foot process effacement (arrows) in Ang II-treated Ksp- $\beta$-cat ${ }^{+/+}$mice compared with Ksp- $\beta$-cat ${ }^{-/-}$mice. Scale bar: 500 nm. (D-F) Scanning electron microscopy (SEM) revealed marked effacement in the control mice compared with untreated control and Ang II-treated Ksp- $\beta$-cat ${ }^{-/-}$mice. Scale bar: $5 \mu \mathrm{m}$.

were increased in Ksp- $\beta$-cat ${ }^{+/+}$, but not Ksp- $\beta$-cat ${ }^{-/}$, kidneys (Figure 4, A and B). MMP-7 gene expression was 3-fold higher in Ksp- $\beta$-cat ${ }^{+/+}$mice compared with that in Ksp- $\beta$-cat ${ }^{-1-}$ mice, although this did not reach significance (Figure 4C, $P=0.092, n=5$ ). MMP-7 protein was localized to renal tubules using immunohistochemistry and again showed greater expression in Ksp- $\beta$-cat ${ }^{+/+}$mice compared with Ksp- $\beta$-cat ${ }^{-/-}$mice or untreated controls (Figure 4D). Based on these results, we hypothesized that MMP-7 plays a role in glomerular injury after chronic Ang II infusion.

Tubule-specific ablation of $\beta$-catenin protects against Adriamycin-induced proteinuria and MMP-7 upregulation. In addition to the Ang II model above, we assessed wild-type and Ksp- $\beta$-cat ${ }^{--}$mice after exposure to Adriamycin, a well-known inducer of proteinuria. We found that Ksp- $\beta$-cat ${ }^{-/-}$mice had significantly less urinary albumin excretion than Ksp- $\beta$-cat ${ }^{+/+}$mice (Figure 5, A and B). Nephrin levels were decreased in Ksp- $\beta$-cat ${ }^{+/+}$mice compared with Ksp- $\beta$-cat ${ }^{\prime-}$ mice (Figure 5C). Further, Ksp- $\beta$-cat ${ }^{\prime-}$ mice expressed less MMP-7, as shown by immunoblot and immunofluorescence (Figure 5, D and E). This demonstrates that the effect of tubular $\beta$-catenin on MMP-7 expression and urinary albumin excretion is generalizable among proteinuric CKD states.

MMP-7 increases glomerular permeability ex vivo and depletes nephrin in glomerular cultures. To provide direct evidence for MMP-7 in mediating glomerular injury, we used an ex vivo glomerular culture model system. To this end, glomeruli were isolated from normal rat kidneys by a sieving method and then exposed to recombinant MMP-7 protein (50 nM). As shown in Figure 6A, MMP-7 treatment clearly increased glomerular permeability ex vivo, leading to an increased release of albumin from the glomerular capillary to surrounding media, in a glomerular permeability assay. This suggests that MMP-7 impairs the integrity of the glomerular filtration barrier. Electron microscopy confirmed that podocyte foot processes became effaced upon addition of MMP-7 to the culture medium (Figure 6B).

We further examined the effect of MMP-7 on the key slit diaphragm protein, nephrin, in cultured glomeruli ex vivo. As shown in Figure 6C, MMP-7 treatment for 30 minutes rapidly decreased nephrin protein abundance in cultured glomeruli. The action of MMP-7 on glomerular nephrin depletion was dose dependent (Figure 6D). We found that nephrin depletion was dependent on the proteolytic activity of MMP-7, since an MMP-7 inhibitor (MMP Inhibitor II) blocked the effect (Figure 6E). Another MMP inhibitor that has no specific activity against MMP-7, GM6001, had no effect on nephrin depletion. These results indicate that MMP-7 can increase glomerular permeability and impair glomerular filtration integrity by degrading and depleting nephrin. 
A

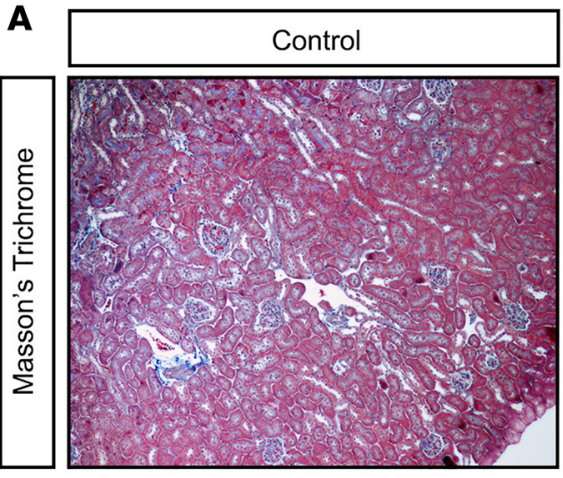

B

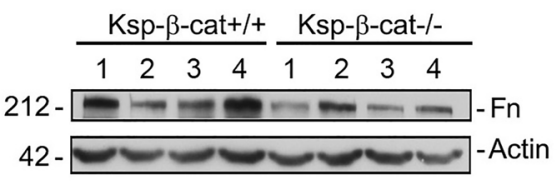

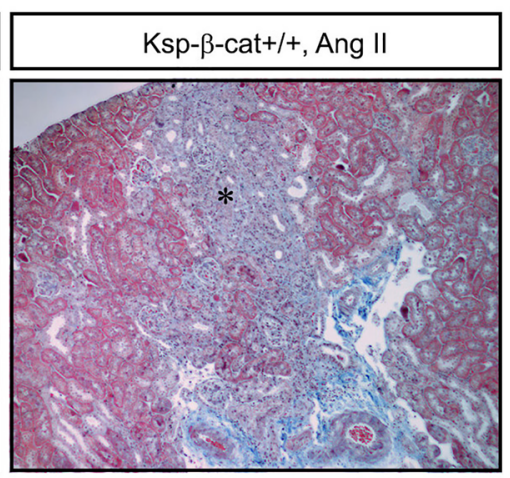
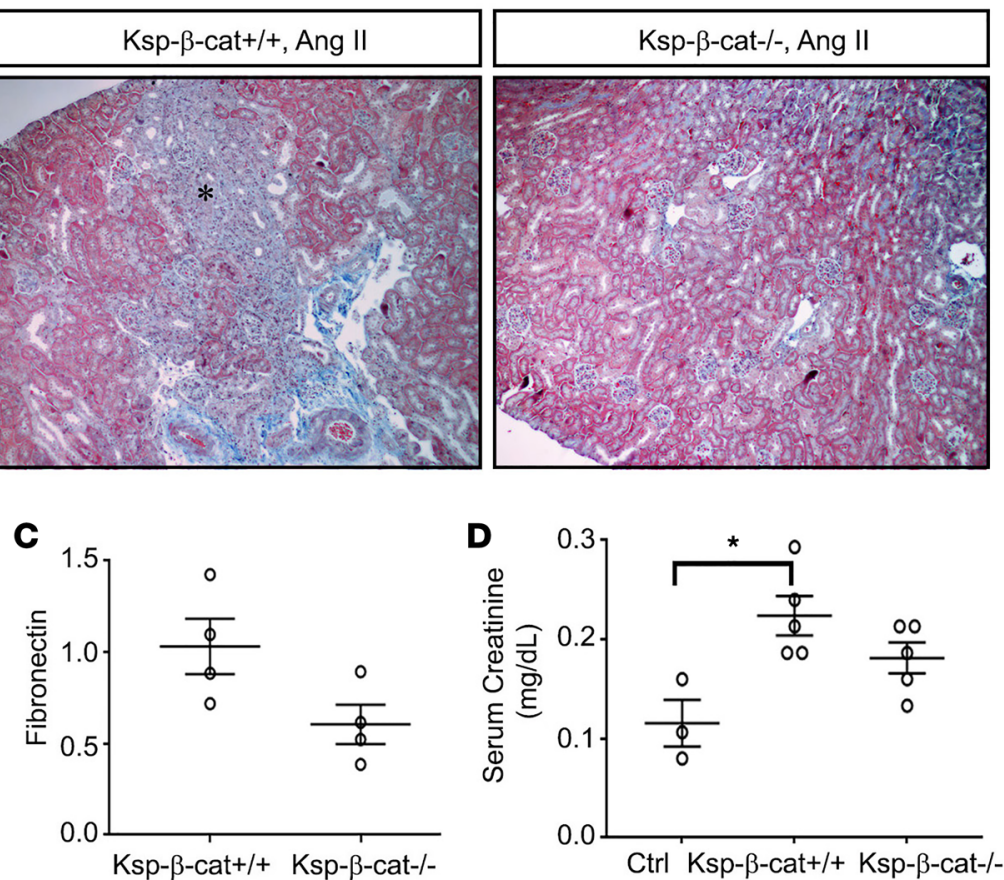

Figure 3. Ablation of $\beta$-catenin in renal tubules reduces interstitial fibrosis induced by Ang II infusion. (A) Masson's trichrome staining showing increased fibrosis in Ksp- $\beta$-cat ${ }^{+/+}$mice after Ang II infusion (asterisk), compared with Ksp- $\beta$-cat ${ }^{-/-}$mice (original magnification, $\left.\times 20\right)$. (B and C) Western blots show a trend toward increased levels of fibronectin in the control mice, compared with Ksp- $\beta$-cat ${ }^{-1-}$ mice $(n=4)$. $P=0.06, t$ test. $(D)$ Measurement of serum creatinine shows that the only significant difference is observed between untreated control mice (Ctrl) and Ksp- $\beta$-cat ${ }^{+/+}$ mice, suggesting moderation of injury in the Ksp- $\beta$-cat ${ }^{-/-}$mice $(n=5) .{ }^{*} P<0.05$, 1 -way ANOVA.

MMP-7 degrades nephrin via proteolytic cleavage. To determine the specificity of the MMP-7 effects on nephrin, isolated rat glomeruli were exposed to different MMPs, including MMP-7, MMP-2, and MMP-9. As shown in Figure 7A, MMP-7 caused nephrin depletion, while both MMP-2 and MMP-9 did not, suggesting specificity of MMP-7 action. We found that only MMP-7, but not the other MMPs, could also deplete podocin, another key component of the slit diaphragm (Figure 7B). Finally, none of the MMPs tested, including MMP-2, MMP-7, and MMP-9, affected other plasma membrane proteins in podocytes, such as integrin $\alpha 3$ and integrin $\beta 1$, suggesting that MMP-7 had a specific effect on slit diaphragm proteins (Figure 7C).

To further examine the effects of MMP-7 on nephrin depletion, we used human embryonic kidney cells (HEK-293 cells) transfected with nephrin expression vector and then incubated with recombinant MMP-7 $(50 \mathrm{nM})$. Nephrin was depleted from HEK-293 cells in a time- and dose-dependent manner (Figure 8, A and B). Degradation also occurred when nephrin was immunoprecipitated from cell lysates and subsequently exposed to MMP-7, and this was blocked effectively by MMP Inhibitor II (Figure 8C), demonstrating that MMP-7 is able to degrade nephrin protein in a cell-free system. To better confirm nephrin degradation by MMP-7, we incubated a truncated form of purified recombinant mouse nephrin protein with MMP-7 (Figure 8D). This truncated form of MMP-7 is $150 \mathrm{kDa}$ in size and contains the majority of the extracellular domains of nephrin (corresponding to Gln37 Thr1049) but lacks the transmembrane segment and cytoplasmic tail (Figure 8E). After incubation with MMP-7, smaller proteolytic fragments of nephrin were detectable in SDS-PAGE (Figure 8D, arrows); this was accompanied by the reduction of 150$\mathrm{kDa}$ truncated nephrin. These experiments indicate that MMP-7 is capable of directly cleaving the nephrin protein, which would lead to slit diaphragm disruption, podocyte injury, and proteinuria.

Exposure to exogenous MMP-7 in vivo induces proteinuria. To provide direct evidence for MMP-7 in mediating podocyte injury in vivo, we examined the effect of MMP-7 overexpression on the integrity of the glomerular filtration barrier in healthy animals. To this end, an MMP-7 expression vector (pCMV-MMP-7) or empty vector (pcDNA3) was injected into normal wild-type mice using a hydrodynamic-based gene transfer approach via tail vein, which leads to transient expression of the transgene (25). We found that plasmid delivery led to upregulation of MMP-7 mRNA in both the liver and the kidney, although the hepatic expression was much 
A

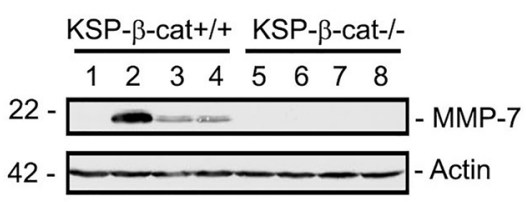

D

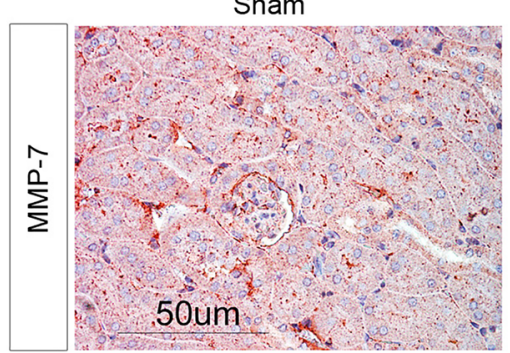

B

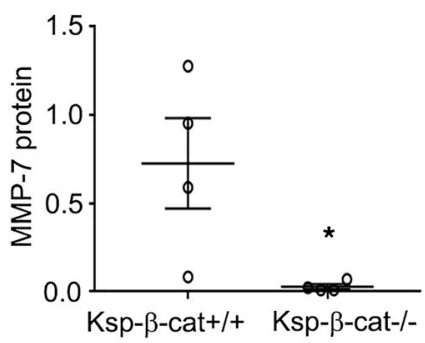

Ksp- $\beta-$ cat+/+

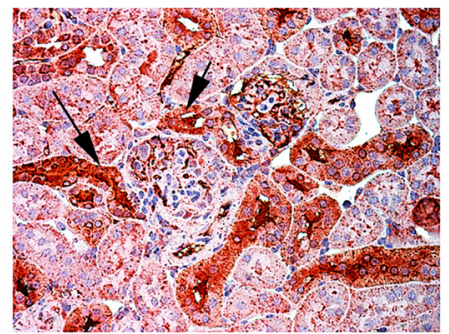

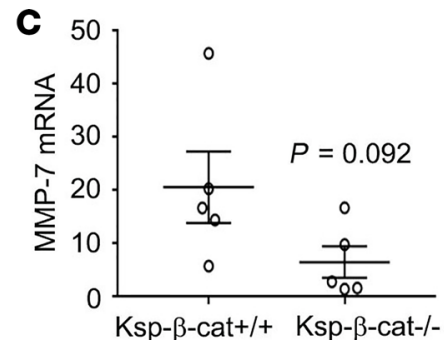

Ksp- $\beta$-cat-/-

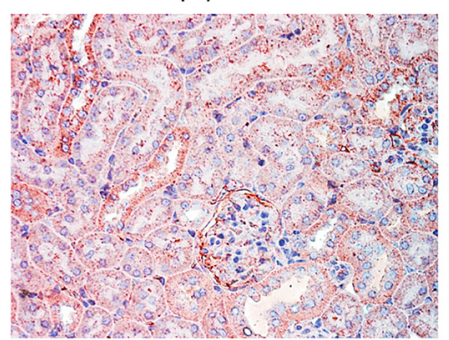

Figure 4. Renal expression of MMP-7 is reduced in Ksp- $\boldsymbol{\beta}-$ cat $^{-/-}$mice after Ang II infusion. (A and B) Western blot analyses for MMP-7 revealed significant reduction in the $\mathrm{Ksp}-\beta$-cat ${ }^{-/-}$mice after Ang II infusion, compared with Ksp- $\beta$-cat - $^{+/+}$mice $(n=4)$. Western blot $(\mathbf{A})$ and quantitation after densitometry (B) are shown. ${ }^{*} P<0.05, t$ test. (C) qRT-PCR shows a trend to higher expression of MMP-7 mRNA in the Ksp- $\beta$-cat ${ }^{+/+}$mice as well $(n=5, t$ test). $P=0.092$, 2-tailed; $P=0.046$, 1-tailed. (D) Immunohistochemical staining for MMP-7 shows negligible staining in control mice, with dramatic upregulation in Ang II-treated Ksp- $\beta$-cat ${ }^{+/+}$mice (arrows). A reduced MMP-7 staining was noticed in Ksp- $\beta$-cat ${ }^{-/-}$mice. Scale bar: $50 \mu \mathrm{M}$.

higher (Supplemental Figure 1, A and B; supplemental material available online with this article; https://doi. org/10.1172/jci.insight.122399DS1). MMP-7 protein could be detected at high levels circulating in the serum (Supplemental Figure 1C). With a molecular weight of $29 \mathrm{kD}$, we expect that it is freely filtered at the glomerulus to gain access to the slit diaphragm. As shown in Figure 9A, MMP-7 expression led to a significant increase in albuminuria. In a similar fashion, injection of recombinant MMP-7 protein $(1 \mathrm{mg} / \mathrm{kg})$ in mice also resulted in an increase in albuminuria, compared with vehicle controls (Figure 9B). These data suggest that elevated MMP-7 is sufficient to induce podocyte injury and impair glomerular filtration.

MMP-7 deficiency protects mice against proteinuria after Ang II infusion. To confirm the role of MMP-7 in mediating podocyte injury after Ang II infusion, we chronically infused Ang II via osmotic minipumps to wild-type $\left(\mathrm{MMP}^{-7^{+/+}}\right)$and MMP-7-null $\left(\mathrm{MMP}^{-7^{-/}}\right)$mice to assess their response to proteinuric injury. As shown in Figure 10A, mice with global ablation of MMP-7 were significantly protected from albuminuria, compared with wild-type controls. These results were further confirmed by gel electrophoresis (Figure 10B). Glomerular nephrin staining was disrupted in wild-type kidneys compared with that in the MMP-7 $7^{-/-}$mice (Figure 10C). MMP-7 $7^{--}$mice were also protected from WT1 depletion by both immunostaining (Figure 10, C and D) and immunoblotting (Figure 10, E and F). Collectively, these data demonstrate that MMP-7 contributes to glomerular/podocyte injury and proteinuria after Ang II infusion.

\section{Discussion}

Treatments for CKD remain extremely limited in scope and efficacy. Since 1993, angiotensin receptor blockers and angiotensin-converting enzyme inhibitors have been the cornerstone of proteinuric CKD treatment, but these agents are only partially effective in reducing proteinuria and often have significant unwanted side effects (26). This problem calls for a better understanding of the exact cellular events leading to progressive CKD with proteinuria. Although it is intuitive to speculate that tubular injury would eventually influence glomerular integrity and health, the underlying mechanism connecting these two events was poorly understood.

The results presented in this study demonstrate that tubule-derived MMP-7 has a profound effect on podocyte biology and glomerular filtration integrity. In mice with tubule-specific ablation of $\beta$-catenin, glomerular integrity is largely preserved after chronic infusion of Ang II. These effects are associated with reduced tubular expression of MMP-7, a robust and direct target of Wnt/ $\beta$-catenin (14). MMP-7 


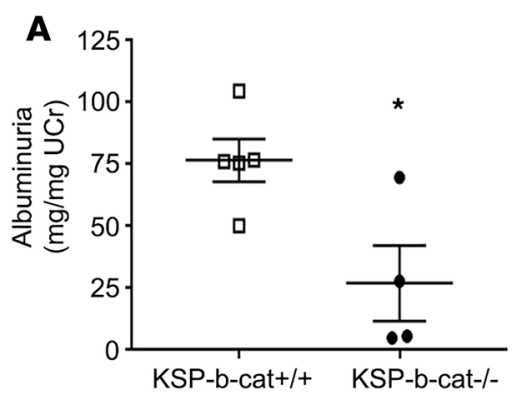

D

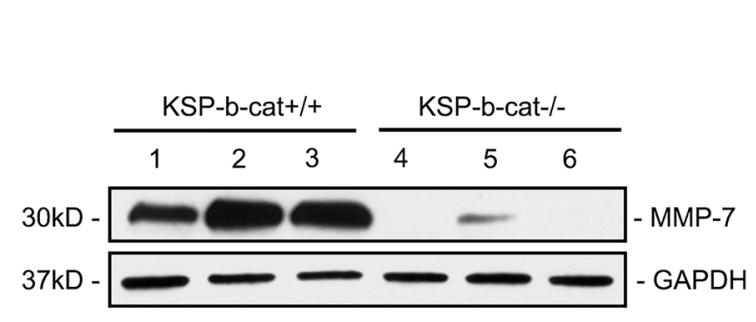

B

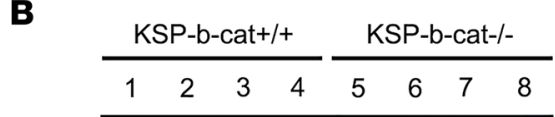

$66 \mathrm{kD}$

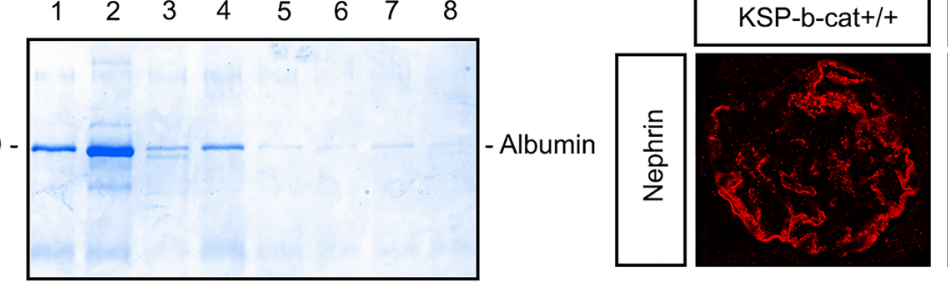

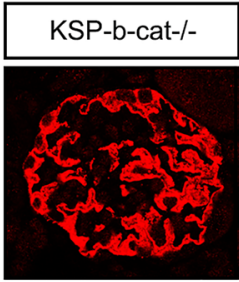

E

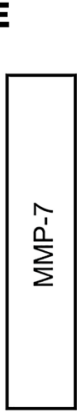

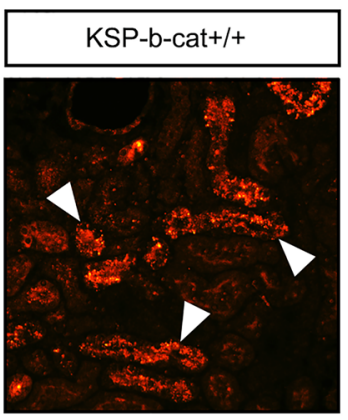

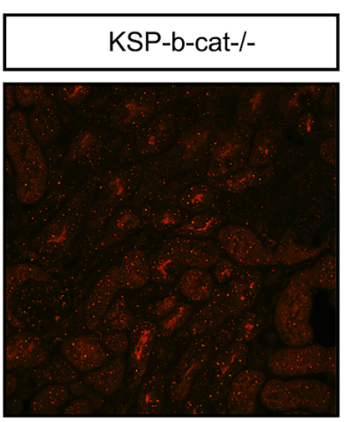

Figure 5. Ksp- $\beta$-cat ${ }^{-1-}$ mice are protected from Adriamycin-induced kidney injury and proteinuria. (A) Urine albumin excretion 3 weeks after injection of Adriamycin $(22 \mathrm{mg} / \mathrm{kg})$ in $\mathrm{KSP}-\beta$-cat ${ }^{+/+}$compared with $\mathrm{KSP}-\beta$-cat ${ }^{-1-}$ mice ${ }^{*} P<0.05, t$ test). (B) Gel electrophoresis of urine samples showing reduction in albumin excretion in KSP- $\beta$-cat ${ }^{-/-}$mice. (C) Immunofluorescence showing greater nephrin disruption in $\mathrm{KSP}^{-} \beta$-cat ${ }^{-/-}$mice (original magnification, $\left.\times 40\right)$. (D) Western blot demonstrating reduced kidney expression of MMP-7 in KSP- $\beta$-cat ${ }^{-/-}$mice. (E) Immunofluorescence for MMP-7 reveals strong upregulation in tubules from KSP- $\beta-\mathrm{cat}^{+/+}$mice only (arrows denote positive staining; original magnification, $\times 20$ ).

directly degrades the key slit diaphragm protein nephrin, as well as podocin, and leads to impairment of glomerular filtration. Therefore, our studies provide evidence for the notion that MMP-7 mediates tubuloglomerular communication in diseased kidneys. Previous studies have shown that there is a complex crosstalk between renal tubular cells and interstitial fibroblasts, leading to the development of fibrosis (27-29). It has also been appreciated that primary glomerular injuries can lead to tubular injury, which is evidenced by the development of tubular atrophy and interstitial fibrosis in a variety of primary glomerular disorders (3). We now propose that the opposite can occur, in that tubule-derived mediators lead to an enhancement of glomerular injury. Tubular-to-glomerular crosstalk should not be surprising, considering the close apposition of renal tubules to glomeruli and the major role of the macula densa in regulating glomerular hemodynamics via nitric oxide and adenosine (30). However, our results are potentially unique in that a tubule-derived protease causes glomerular injury by direct degradation of the key slit diaphragm proteins in podocytes.

The means by which a soluble protease reaches the glomerulus requires further study but could theoretically occur through several pathways, including diffusion from nearby tubules. Further, it is well known that tubular injury can lead to tubular apoptosis, loss of barrier integrity, and the potential backleak of substances through injured tubules, which could affect glomeruli (31-34). Finally, it is possible that MMP-7 enters the systemic circulation. At $29 \mathrm{kDa}$, we would expect that some amount of MMP-7 could be freely filtered at the glomerulus to gain access to the slit diaphragm. These potential mechanisms require further exploration.

MMP-7 is a matrix metalloprotease that is highly upregulated in renal tubules during kidney injury. MMP-7 is increased in experimental unilateral ureteral obstruction, acute kidney injury, polycystic kidney disease, and Adriamycin nephropathy as well as in human kidney biopsies obtained from various CKD patients and in human urine after injury $(14,15,35)$. It is a known target for $\beta$-catenin and its promoter contains 2 binding sites for the $\beta$-catenin transcription cofactor T cell factor (TCF). MMP-7 is associated with and stimulated by canonical Wnt signaling or $\beta$-catenin and can be blocked by Wnt or $\beta$-catenin inhibition (14). Our studies using Ang II infusion (Figure 4) and Adriamycin (Figure 5) demonstrate that Ksp- $\beta$-cat ${ }^{-/-}$mice have reduced MMP-7 expression after injury, in agreement with prior studies using other experimental kidney injuries (36). The induction of MMP-7 in a wide variety of CKD models is consistent 


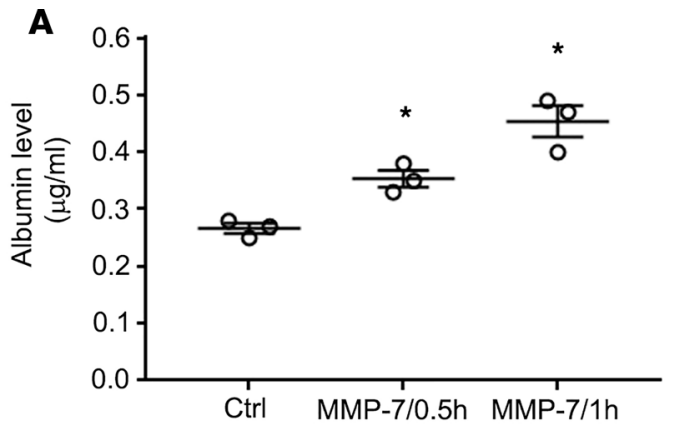

C

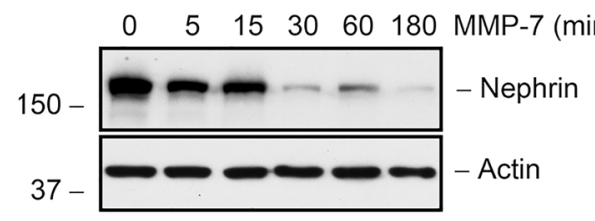

B

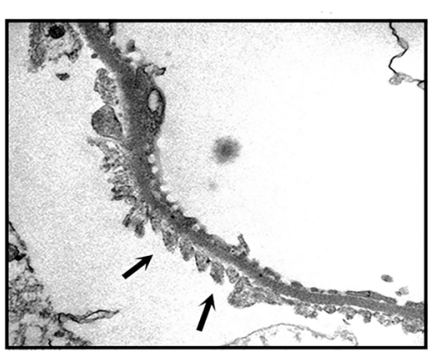

MMP-7

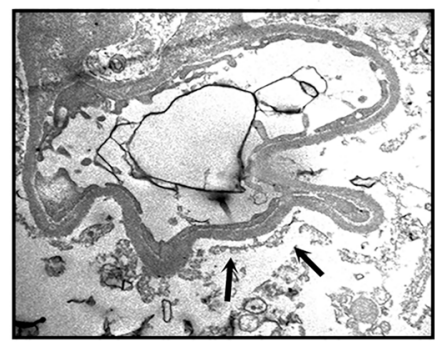

D
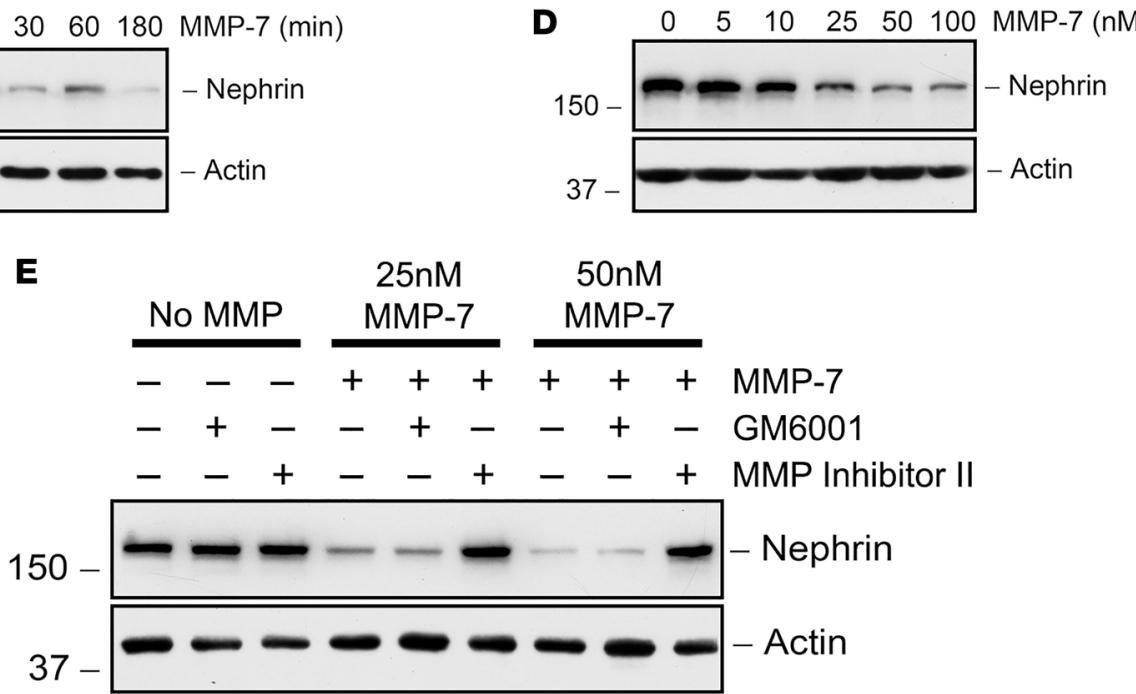

Figure 6. Recombinant MMP-7 decreases nephrin in a time- and dose-dependent manner in ex vivo glomeruli. (A) MMP-7 treatment (50 nM, 30 min) causes a significant time-dependent release of albumin from isolated rat glomeruli. ${ }^{*} P<0.05$, repeated-measures 1 -way ANOVA. (B) TEM of isolated glomeruli shows flattening and fusion of foot processes in MMP-7-treated glomeruli compared with vehicle controls (arrows) (original magnification, $\times 20,000$ ). In separate experiments, glomeruli were incubated with either the same concentration of MMP-7 (50 nM) for various periods of time (C) or with increasing amounts of MMP-7 for 60 minutes (D). Clomerular lysates were immunoblotted with antibodies against nephrin and actin. (E) Nephrin depletion by MMP-7 is dependent on its proteolytic activity. Rat glomeruli were pretreated with GM6001 (25 $\mu$ M) or MMP Inhibitor II (20 $\mu$ M) for 30 minutes before incubation with MMP-7 (25 nM or $50 \mathrm{nM}$ ) for 60 minutes.

with the notion that glomerular injury and proteinuria are an inevitable consequence of perhaps all $\mathrm{CKD}$, regardless of the initial etiologies.

Besides matrix proteins, the substrates of MMP-7 include several extracellular, nonmatrix proteins, such as E-cadherin, Fas ligand, defensins, and other MMPs (14, 36, 37). For the first time to our knowledge, we now demonstrate that nephrin is a specific and direct substrate of MMP-7. This conclusion is supported by several lines of evidence. First, MMP-7 can degrade nephrin in cultured glomeruli, cultured cells, and cell-free systems, which is dependent on its proteolytic activity. Second, the action of MMP-7 on nephrin degradation was rapid, starting as early as 5 minutes after incubation (Figure 6). Third, the action of MMP-7 on nephrin was direct, cleaving purified recombinant nephrin protein in a cell-free system (Figure 8). Finally, the action of MMP-7 was specific, as other MMPs, such as MMP-2 and MMP-9, are unable to degrade nephrin in the same conditions. Notably, MMP-7 also decreased levels of podocin in cultured glomeruli (Figure 7), suggesting it cleaves podocin as well, although it had no effect on other podocyte membrane proteins, including integrin $\alpha 3$ and integrin $\beta 1$. However, we cannot exclude the possibility that the decrease of podocin in cultured glomeruli after MMP-7 incubation could be a result of nephrin loss, because nephrin/podocin often form complexes and stabilize each other. Regardless of the mechanism involved, the loss of key slit diaphragm proteins, such as nephrin and podocin, would be sufficient, by themselves, to increase glomerular permeability and impair glomerular filtration (38). Consistent with this view, exogenous MMP-7 is sufficient to induce proteinuria in mice (Figure 9). Although the effects of injected MMP-7 are transient in mouse model, we would 
A

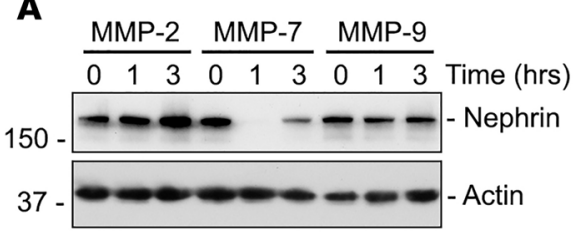

B

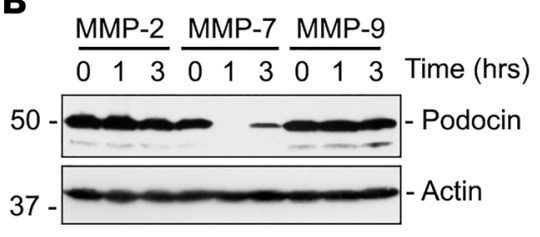

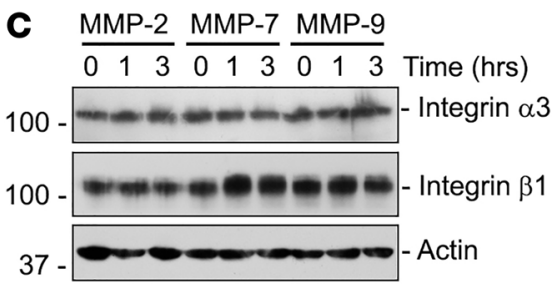

Figure 7. MMP-7 reduces nephrin and podocin levels in isolated glomeruli. (A) Rat glomeruli were incubated separately with MMP-2 (1 $\mu \mathrm{g} / \mathrm{ml}$ or $13.88 \mathrm{nM})$, MMP-7 (50 nM), and MMP-9 $(1 \mu \mathrm{g} / \mathrm{ml}$ or $10.9 \mathrm{nM})$ and glomerular lysates were immunoblotted for nephrin. Only MMP-7 was capable of reducing nephrin levels. (B) MMP-7 also reduced podocin protein in isolated glomeruli ex vivo. Glomerular lysates were immunoblotted with antibodies against podocin and actin. (C) MMP-7 did not affect other membrane proteins in podocytes, such as integrin $\alpha 3$ and $\beta 1$. Western blot analyses of glomerular lysates reveal no effect on integrin $\alpha 3$ and $\beta 1$ proteins after incubation with any of the 3 MMPs.

anticipate that, in CKD settings, MMP-7 is produced chronically and continuously and therefore would lead to detrimental effects on the integrity of glomerular filtration (15).

MMP-7 $7^{-1}$ mice were also protected against proteinuric kidney disease (Figure 10). We must acknowledge that mice with global deficiency of MMP-7 cannot definitively prove the involvement of tubular MMP-7 in glomerular disease, but tubular-specific MMP-7-/- mice unfortunately do not exist. Nevertheless, the work with MMP- $7^{-/-}$mice provides further support of the importance of this protease in proteinuria.

It should be pointed out that Ksp- $\beta$-cat ${ }^{-1-}$ mice are also protected from the Ang II-induced development of interstitial fibrosis (Figure 3). This is not surprising, as earlier studies have shown that $\beta$-catenin-driven MMP-7 is a pathogenic mediator of kidney fibrosis by degrading E-cadherin, which leads to $\beta$-catenin liberation and activation, forming a vicious cycle (16). Furthermore, this reduction of fibrosis in Ksp- $\beta$-cat ${ }^{--}$mice after Ang II infusion could be a result of the diminished glomerular injury and proteinuria, because proteinuria per se aggravates tubulointerstitial lesions as described previously $(39,40)$. In addition, the activation of tubular $\beta$-catenin after Ang II infusion could have a number of MMP-7-independent effects that promote interstitial fibrosis, including effects on the expression of

A

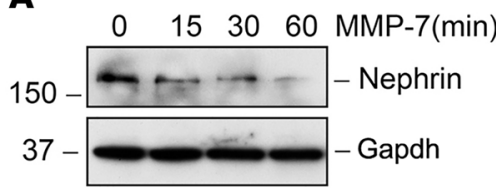

D

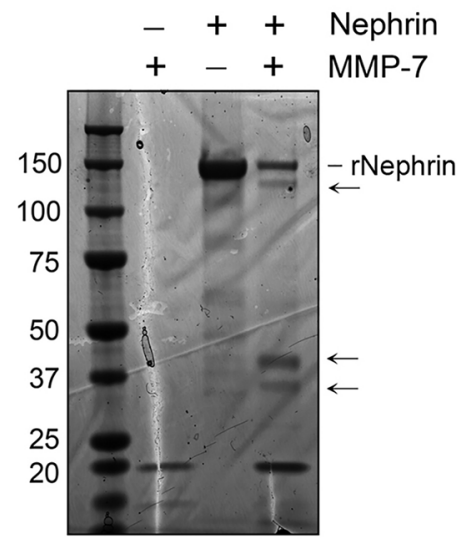

B

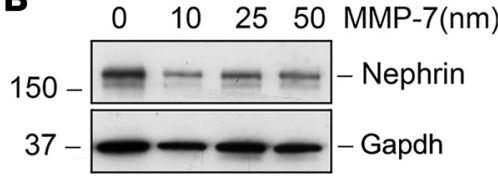

E

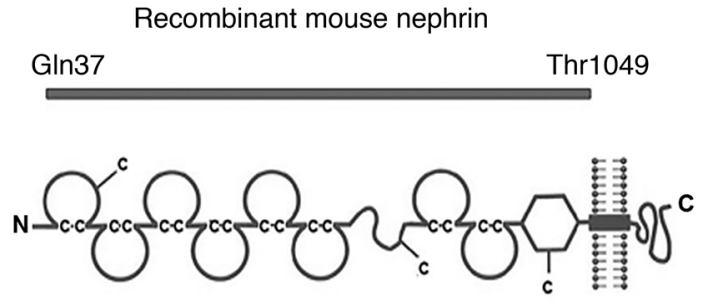

Figure 8. MMP-7 mediates nephrin degradation in vitro. (A and B) HEK-293 cells were transfected with nephrin expression vector for 48 hours, followed by incubation with MMP-7 (50 nM) for various periods of time (A) or with increasing amounts of MMP-7 for 60 minutes (B). (C) Nephrin from overexpressing cells was immunoprecipitated with anti-nephrin antibody, followed by various treatments as indicated. MMP-7 caused nephrin depletion, which was inhibited by MMP Inhibitor II. ( $D$ and E) Representative SDS-PAGE shows that MMP-7 degraded recombinant mouse nephrin protein. Purified mouse nephrin protein $(2 \mu \mathrm{g}$ ) was incubated with $50 \mathrm{nM}$ MMP-7 for 60 minutes. Truncated form of mouse nephrin corresponding to GIn37 to Thr1049 ( 150 kDa in size) is used (E). Arrows in $\mathbf{D}$ indicate the degradation fragments of nephrin. 

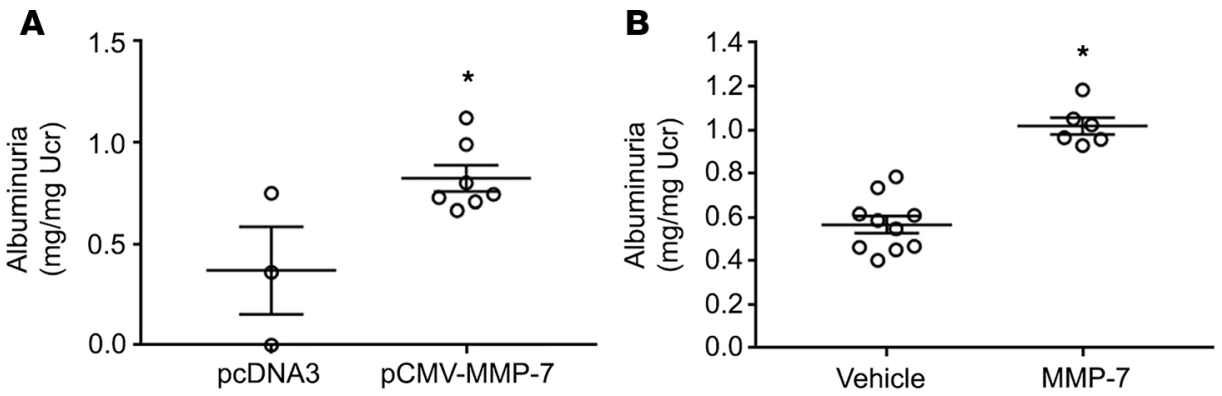

Figure 9. Delivery of MMP-7 gene or recombinant MMP-7 protein induces albuminuria in vivo. (A) Mice were injected intravenously with pcDNA3 or pCMV-MMP-7 vector $(2 \mathrm{mg} / \mathrm{kg})$, respectively. Urinary albumin concentration was assayed 24 hours after injection. ${ }^{*} P<0.05$ ( $n=7, t$ test). (B) After injection with recombinant MMP-7 (1 $\mathrm{mg} / \mathrm{kg}$ ) for 3 hours, urinary albumin excretion was increased in the MMP-7-treated group, compared with vehicle controls. ${ }^{*} P<0.05(n=6, t$ test $)$.

the renin-angiotensin system, Snail1, and fibronectin $(6,7,41)$. Therefore, tubular $\beta$-catenin signaling during AngII exposure could, through multiple pathways, lead to progressive interstitial fibrosis that is characteristic of all forms of CKD.

Overall, these studies uncover a potentially novel mechanism to account for how tubular injury triggers glomerular damage during CKD progression. We show that kidney injuries after Ang II infusion or Adriamycin exposure lead to tubular $\beta$-catenin activation, which in turn upregulates soluble MMP-7 expression in renal tubules. Such tubule-derived MMP-7 causes deleterious glomerular changes, including the cleavage of nephrin, and result in glomerular filtration impairment and higher urinary protein excretion. This would lead to further tubular $\beta$-catenin activation and MMP-7 expression in a positive feedback loop that ultimately contributes to progressive renal failure and fibrosis. Therapies directed against $\beta$-catenin and MMP-7 could be useful in the treatment of CKD.

\section{Methods}

Animals and treatment protocol. Ksp- $\beta$-cat ${ }^{-1-}$ mice were generated as previously described and are on the C57BL/ 6 background strain (18). Ksp- $\beta$-cat ${ }^{+/+}$controls were $\beta$-catenin-floxed, Cre-negative littermates. Mice were 8-10 weeks of age at initiation of the experiments, and age and sex were equally distributed in both groups. MMP-7//- mice and wild-type C57BL/6J mice were obtained from The Jackson Laboratory (stock 005111 and 000664).

To sensitize mice to the Ang II model, a unilateral nephrectomy was performed on day -7 . On day 0 , an osmotic minipump (Alzet) was implanted subcutaneously. The minipump delivered $1.5 \mathrm{mg} / \mathrm{kg} / \mathrm{d}$ Ang II (Bachem) as previously described (19). Urine was collected weekly until sacrifice, at which time the kidneys, serum, and final urine sample were collected. Adriamycin (MilliporeSigma) injury was induced with a single intravenous dose of $22 \mathrm{mg} / \mathrm{kg}$ in nonnephrectomized mice. This high dose of Adriamycin is necessary to induce injury in the resistant C57BL/6 strain and was titrated empirically prior to our experiment $(42,43)$.

Histology. For histologic assessment, kidneys were fixed in $10 \%$ buffered formalin, embedded in par-

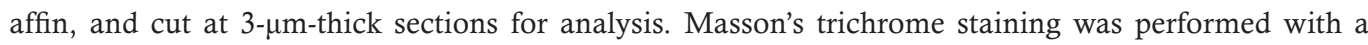
Masson's trichrome kit from Thermo Fisher Scientific.

Renal function measurements. Serum creatinine was determined with a Creatinine Enzymatic Kit (catalog no. C7548, Pointe Scientific) using an enzymatic method that is superior to Jaffe assays and compares favorably with HPLC methods (44). Urinary albumin excretion (albuminuria) was detected by use of a mouse albumin ELISA kit (Bethyl Laboratories). Urinary albumin was normalized to the urine creatinine, which was determined using the same creatinine kit noted above. Urine samples were loaded by equivalent urinary creatinine levels into polyacrylamide gels and subjected to electrophoresis prior to staining with Coomassie blue.

Immunofluorescence. Frozen kidney samples were embedded at sacrifice in OCT medium (Scigen Scientific), and sections were cut on a microtome at 3- $\mu \mathrm{m}$ thickness. Sections were then fixed in $4 \%$ paraformaldehyde, followed by permeabilization in $0.2 \%$ Triton in PBS. Sections were blocked with normal 


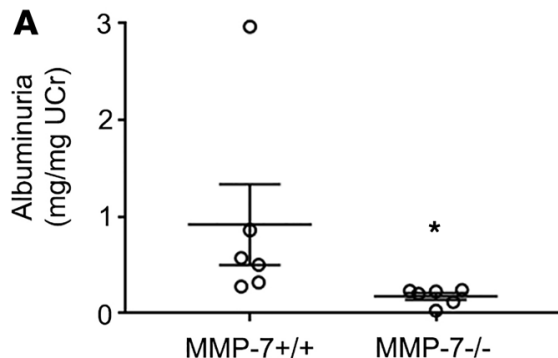

C
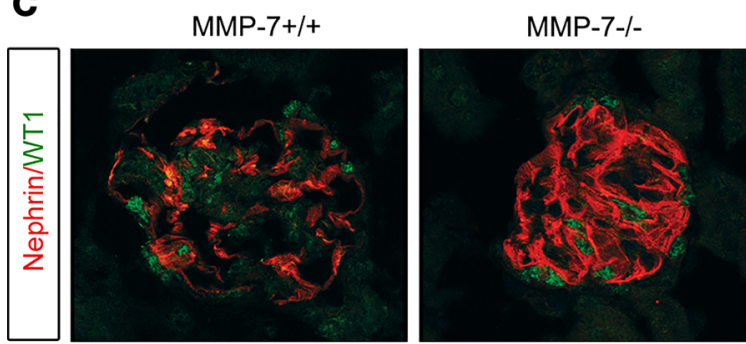

$\mathbf{E}$

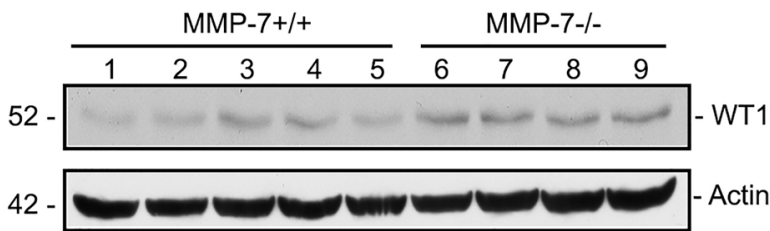

B

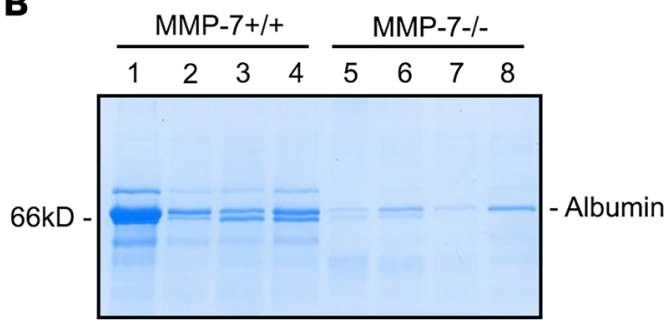

D

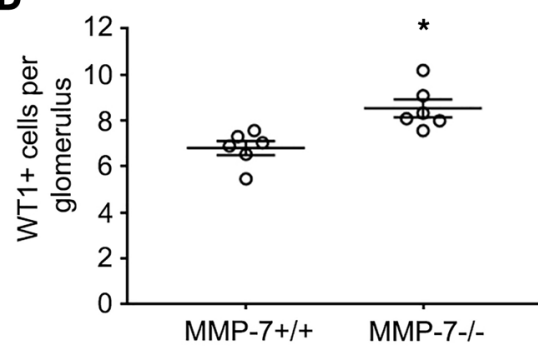

$\mathbf{F}$

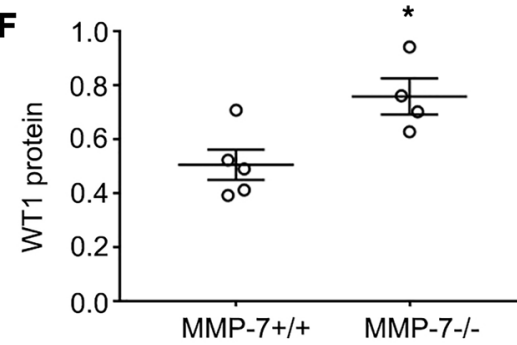

Figure 10. Mice with global ablation of MMP-7 are protected from Ang II-induced glomerular injury. Wild-type and MMP-7/- knockout mice were treated with continuous infusions of Ang II $(1.5 \mathrm{mg} / \mathrm{kg} / \mathrm{d})$. (A) Albuminuria is significantly reduced in the knockout compared with wild-type mice. (B) Gel electrophoresis of urine samples demonstrates a reduced albumin excretion in MMP-- ${ }^{-/-}$mice, compared with MMP- $7^{+/+}$counterparts. (C) Immunofluorescence staining shows extensive nephrin disruption and a decrease in numbers of healthy WT1-positive podocytes in MMP-7+/+ mice. MMP-7-knockout mice retained linear nephrin staining and numbers of WT1-positive nuclei (original magnification, $\times 40)$. (D) Quantitation of WT1-positive nuclei per glomerulus $(n=5-6, t$ test). (E and $\mathbf{F})$ Western blot analyses show an increased preservation of WT1 expression in MMP-7 $7^{-/-}$mice after Ang II infusion, compared with MMP-7 ${ }^{+/+}$controls. Western blot $(\mathbf{E})$ and quantitation $(\mathbf{F})$ are shown. ${ }^{*} P<0.05(n=4-5, t$ test $)$.

serum and then incubated overnight in primary antibody for nephrin (2OR-NP002; Fitzgerald Industries) and WT1 (sc-192; Santa Cruz Biotechnology). FITC- and Cy3-conjugated secondary antibodies (Jackson ImmunoResearch Laboratories) were used for detection. Sections were visualized on a Leica SP5 CW-STED confocal microscope.

To determine the percentage of glomeruli with disrupted nephrin, at least 20 glomeruli for each animal were evaluated. Any breaks in the normal linear nephrin staining were counted as positive for disruption. For WT1-positive cells, at least 20 glomeruli were evaluated for each animal. The number of WT1-positive cells per glomerulus were counted and averaged per animal, and these numbers were averaged for a group mean.

Immunohistochemistry. Three-micron paraffin-embedded sections were deparaffinized and rehydrated through graded ethanol $(100 \%, 95 \%$, and $70 \%)$ to distilled water and subjected to antigen retrieval with 10 $\mathrm{mM}$ sodium citrate ( $\mathrm{pH}$ 6.0) buffer for 30 minutes. Sections were then incubated with $3 \% \mathrm{H}_{2} \mathrm{O}_{2}$ to block endogenous peroxidase activity, permeabilized in PBS-Tween, and blocked in 3\% BSA before overnight incubation with anti-cubilin (sc-25470, Santa Cruz Biotechnology), anti-megalin (sc-20609, Santa Cruz Biotechnology), and anti-MMP-7 antibodies (GTX11716, Genetex). Immunohistochemical staining was performed according to the established protocol as described previously (19). 
Immunoblotting. Kidneys were homogenized in RIPA buffer containing a protease inhibitor cocktail (78442, Thermo Fisher Scientific), and concentration was determined with BCA protein assay (Thermo Fisher Scientific). Gel electrophoresis was performed on reduced, denatured samples. After blotting onto PVDF membranes (GE Healthcare Life Sciences) and blocking with 5\% milk, membranes were incubated with primary antibodies. HRP-conjugated secondary antibodies (goat anti-mouse, catalog A16072; goat anti-rabbit, catalog A16104; Invitrogen) and Supersignal West Pico substrate (Thermo Fisher Scientific) were used for detection. Densitometry was performed using ImageJ software (NIH). The following primary antibodies were used: MMP-7 (GTX11716, Genetex), fibronectin (F3648, MilliporeSigma), actin (MAB1501, MilliporeSigma), WT1 (sc-192, Santa Cruz Biotechnology), integrin $\alpha 3$ and integrin $\beta 1$ (611044 and 610468, BD Biosciences). See complete unedited blots in the supplemental material.

qRT-PCR. RNA was isolated from kidney tissue homogenates using TRIzol reagent (Thermo Fisher Scientific). A reverse transcriptase reaction was performed to obtain $\mathrm{CDNA}$, and this was used in a quantitative, real-time reverse transcriptase PCR (qRT-PCR) reaction using SYBR green in a CFX Connect instrument (Bio-Rad). The primer sequences to detect mouse MMP-7 were 5'-TAGGCGGAGATGCTCACTTT-3' (forward) and 5'-TTCTGAATGCCTGCAATGTC-3' (reverse), while human MMP-7 (for detection of plasmid derived MMP-7) was detected with 5'-TTGATGGGCCAGGAAACACG-3' (forward) and 5'-CTACCATCCGTCCAGCGTTC-3' (reverse). Melt curves were used to ensure the specificity of the PCR product. Data were analyzed using the $2^{-\Delta \Delta C t}$ method (45).

MMP-7 ELISA. An ELISA for human total MMP-7 was performed on serum samples from mice injected with human MMP-7 plasmid following the manufacturer's instructions (DMP700, R\&D Systems).

Ex vivo glomerular cultures. To obtain primary rat glomeruli for culture, adult male Sprague Dawley rats (Envigo) were anesthetized, and their kidneys were removed. Each kidney was carefully minced and passed through 3 sequentially smaller sieves to remove renal tubules and allow glomeruli to pass through per a previously described protocol (46). The flow-through was centrifuged to pellet the glomeruli, and this was resuspended in RPMI-1640 with 10\% FBS and $100 \mathrm{U} / \mathrm{ml}$ penicillin/streptomycin. MMP-2, MMP-7, and MMP9 (R\&D Systems) and GM6001 and MMP Inhibitor II (both from EMD Millipore) were added as described.

To determine albumin permeability, we performed experiments as previously described (47). Briefly, ex vivo glomeruli were incubated in media containing 5\% BSA (MilliporeSigma). Recombinant MMP-7 was then added and incubated for 30 minutes. Glomeruli were then washed and incubated in low BSA media, and samples were collected at 30 minutes and 1 hour. The BSA content of this media was determined by ELISA (Bethyl Laboratories).

MMP-7 plasmid. An active mutant human MMP-7 clone with a substitution of valine to glycine at amino acid 92 was provided by Carole L. Wilson (Medical University of South Carolina, Charleston, South Carolina, USA). The full-length coding sequence of MMP-7 cDNA was amplified by using a standard RT-PCR protocol. The PCR product was cloned into the mammalian expression vector p3XFlag-CMV (MilliporeSigma) and confirmed by DNA sequencing at the University of Pittsburgh Biomedical Research Support Facilities. Plasmid was injected into mice via tail vein at a dose of $2 \mathrm{mg} / \mathrm{kg}$ plasmid in a $2-\mathrm{mL}$ volume delivered within a 10-second time period, as adapted from prior published protocols (25).

TEM. TEM and SEM were performed as previously described (48). Portions of mouse kidneys were immersion fixed with 2.5\% glutaraldehyde in PBS for more than 24 hours. Several $1-\mathrm{mm}^{3}$ cubes were obtained, washed 3 times in PBS, and then post-fixed in aqueous $1 \% \mathrm{OsO}_{4}, 1 \% \mathrm{~K}_{3} \mathrm{Fe}(\mathrm{CN})_{6}$ for 1 hour. Following 3 PBS washes, the pellet was dehydrated through a graded series of $30 \%-100 \%$ ethanol; $100 \%$ propylene oxide was then infiltrated in 1:1 mixture of propylene oxide/Polybed 812 epoxy resin (Polysciences) for 1 hour. After several changes of $100 \%$ resin over 24 hours, the pellet was embedded in molds, cured at $37^{\circ} \mathrm{C}$ overnight, followed by additional hardening at $65^{\circ} \mathrm{C}$ for 2 more days. Ultrathin $(60 \mathrm{~nm})$ sections were cut on a Leica EM UC7 ultramicrotome, collected on 200 mesh copper grids, and stained with $4 \%$ uranyl acetate for 10 minutes, followed by $1 \%$ lead citrate for 7 minutes using the Leica EM AC20 automatic stainer. Sections were imaged using a JEOL JEM 1011 transmission electron microscope at $80 \mathrm{kV}$ fitted with a side mount AMT $2 \mathrm{k}$ digital camera (Advanced Microscopy Techniques).

SEM. Kidney tissue was processed as for TEM above, but 1-mm-thick longitudinal slices were used instead of cubes. Tissue was processed up to the final 100\% ethanol and then chemically dried using hexamethyldisilazane. Dried slices were mounted onto aluminum stubs, grounded with silver paint, and then sputter coated with $3.5 \mathrm{~nm}$ gold/palladium (Auto 108, Cressington). Samples were viewed in a JEOL JSM-6330F scanning electron microscope at $3 \mathrm{kV}$. 
Statistics. Data are presented as mean \pm SEM. Student's $t$ test (2-tailed) was used for comparisons between 2 groups, while a 1 -way ANOVA was used for more than 2 groups. $P<0.05$ was the threshold used for significance. Statistical tests were run on GraphPad Prism.

Study approval. All studies using animals were performed to the ethical and scientific standards recommended by the Guide for the Care and Use of Laboratory Animals (National Academies Press, 2011) of the NIH. The animal protocol was approved by the Institutional Animal Care and Use Committee at the University of Pittsburgh.

\section{Author contributions}

RJT, Y. Li, BMR, DMC, DZ, JH, DBS, and Y. Liu designed the experiments. RJT, Y. Li, BMR, DMC, HF, and DZ performed the experiments. RJT, Y. Li, BMR, DMC, DZ, DBS, and Y. Liu analyzed the results. RJT and Y. Liu wrote the manuscript. All authors reviewed the final version of the manuscript.

\section{Acknowledgments}

This work was supported by funding from NIH grants R01DK064005 and R01DK106049 (to Y. Liu), R01DK103776 (to JH), and P30DK079307 (Pittsburgh Center for Kidney Research) and National Science Foundation of China grant 81521003. RJT was supported by NIH grant T32DK061296, an American Heart Association Award (13FTF160990086), an American Society of Nephrology Carl W. Gottschalk Research Scholarship Grant, a University of Pittsburgh Medical Center Competitive Medical Research Fund, and a University of Pittsburgh Physicians Academic Foundation Award. DMC was supported by a Nephrotic Syndrome Study Network Career Development Fellowship. DZ is supported by NIH grant K01DK116816.

Address correspondence to: Roderick Tan, Department of Medicine, Renal-Electrolyte Division, 828A Scaife Hall, 3550 Terrace Street, Pittsburgh, Pennsylvania 15261, USA. Phone: 412.624.4008; Email: tanrj@upmc.edu. Or to: Youhua Liu, Department of Pathology, University of Pittsburgh, S-405 Biomedical Science Tower, 200 Lothrop Street, Pittsburgh, Pennsylvania 15261, USA. Phone: 412.648.8253; Email: yhliu@pitt.edu.

1. CDC. Chronic Kidney Disease Initiative. http://www.cdc.gov/ckd. Updated July 22, 2019. Accessed November 18, 2019.

2. Abbate M, Zoja C, Remuzzi G. How does proteinuria cause progressive renal damage? J Am Soc Nephrol. 2006;17(11):2974-2984

3. Kriz W, LeHir M. Pathways to nephron loss starting from glomerular diseases-insights from animal models. Kidney Int. 2005;67(2):404-419.

4. Carlström M, Wilcox CS, Arendshorst WJ. Renal autoregulation in health and disease. Physiol Rev. 2015;95(2):405-511.

5. Grgic I, et al. Targeted proximal tubule injury triggers interstitial fibrosis and glomerulosclerosis. Kidney Int. 2012;82(2):172-183.

6. Carlisle RE, et al. TDAG51 mediates epithelial-to-mesenchymal transition in human proximal tubular epithelium. Am J Physiol Renal Physiol. 2012;303(3):F467-F481.

7. DiRocco DP, Kobayashi A, Taketo MM, McMahon AP, Humphreys BD. Wnt4/ $\beta$-catenin signaling in medullary kidney myofibroblasts. J Am Soc Nephrol. 2013;24(9):1399-1412.

8. Kawakami T, Ren S, Duffield JS. Wnt signalling in kidney diseases: dual roles in renal injury and repair. J Pathol. 2013;229(2):221-231

9. Lin SL, et al. Macrophage Wnt7b is critical for kidney repair and regeneration. Proc Natl Acad Sci USA. 2010;107(9):4194-4199.

10. Edeling M, Ragi G, Huang S, Pavenstädt H, Susztak K. Developmental signalling pathways in renal fibrosis: the roles of Notch, Wnt and Hedgehog. Nat Rev Nephrol. 2016;12(7):426-439.

11. Maarouf $\mathrm{OH}$, et al. Paracrine Wnt1 drives interstitial fibrosis without inflammation by tubulointerstitial cross-talk. $J A m$ Soc Nephrol. 2016;27(3):781-790.

12. Xiao L, et al. Sustained activation of Wnt/ $\beta$-catenin signaling drives AKI to CKD progression. J Am Soc Nephrol. 2016;27(6):1727-1740.

13. Zhou L, et al. Multiple genes of the renin-angiotensin system are novel targets of Wnt/ $\beta$-catenin signaling. J Am Soc Nephrol. 2015;26(1):107-120.

14. He W, et al. Matrix metalloproteinase-7 as a surrogate marker predicts renal Wnt/ $\beta$-catenin activity in CKD. J Am Soc Nephrol. 2012;23(2):294-304

15. Surendran K, Simon TC, Liapis H, McGuire JK. Matrilysin (MMP-7) expression in renal tubular damage: association with Wnt4. Kidney Int. 2004;65(6):2212-2222.

16. Zhou D, et al. Matrix metalloproteinase-7 is a urinary biomarker and pathogenic mediator of kidney fibrosis. $J$ Am Soc Nephrol. 2017;28(2):598-611.

17. Yang X, et al. Urinary matrix metalloproteinase-7 predicts severe AKI and poor outcomes after cardiac surgery. $J$ Am Soc Nephrol. 2017;28(11):3373-3382.

18. Zhou D, Li Y, Lin L, Zhou L, Igarashi P, Liu Y. Tubule-specific ablation of endogenous $\beta$-catenin aggravates acute kidney injury 
in mice. Kidney Int. 2012;82(5):537-547.

19. Tan RJ, et al. Extracellular superoxide dismutase protects against proteinuric kidney disease. J Am Soc Nephrol. 2015;26(10):2447-2459.

20. Zhou Y, et al. Induction of cytochrome P450 4A14 contributes to angiotensin II-induced renal fibrosis in mice. Biochim Biophys Acta Mol Basis Dis. 2018;1864(3):860-870.

21. Eckel J, et al. TRPC6 enhances angiotensin II-induced albuminuria. J Am Soc Nephrol. 2011;22(3):526-535.

22. Cechova S, Dong F, Chan F, Kelley MJ, Ruiz P, Le TH. MYH9 E1841K Mutation augments proteinuria and podocyte injury and migration. J Am Soc Nephrol. 2018;29(1):155-167.

23. Kandasamy Y, Smith R, Lumbers ER, Rudd D. Nephrin - a biomarker of early glomerular injury. Biomark Res. $2014 ; 2: 21$.

24. Zhou L, et al. Mutual antagonism of Wilms' tumor 1 and $\beta$-catenin dictates podocyte health and disease. J Am Soc Nephrol. 2015;26(3):677-691

25. Liu F, Song Y, Liu D. Hydrodynamics-based transfection in animals by systemic administration of plasmid DNA. Gene Ther. 1999;6(7):1258-1266.

26. Johnson SA, Spurney RF. Twenty years after ACEIs and ARBs: emerging treatment strategies for diabetic nephropathy. Am J Physiol Renal Physiol. 2015;309(10):F807-F820.

27. Fabian SL, et al. Hedgehog-Gli pathway activation during kidney fibrosis. Am J Pathol. 2012;180(4):1441-1453.

28. Gewin L, Zent R, Pozzi A. Progression of chronic kidney disease: too much cellular talk causes damage. Kidney Int. 2017;91(3):552-560.

29. Qi R, Yang C. Renal tubular epithelial cells: the neglected mediator of tubulointerstitial fibrosis after injury. Cell Death Dis 2018;9(11):1126.

30. Komlosi P, Bell PD, Zhang ZR. Tubuloglomerular feedback mechanisms in nephron segments beyond the macula densa. Curr Opin Nephrol Hypertens. 2009;18(1):57-62.

31. Xiong C, et al. Pharmacological inhibition of Src kinase protects against acute kidney injury in a murine model of renal ischemia/reperfusion. Oncotarget. 2017;8(19):31238-31253.

32. Wang S, et al. Massive proteinuria-induced injury of tubular epithelial cells in nephrotic syndrome is not exacerbated by furosemide. Cell Physiol Biochem. 2018;45(4):1700-1706.

33. Morais C, Westhuyzen J, Metharom P, Healy H. High molecular weight plasma proteins induce apoptosis and Fas/FasL expression in human proximal tubular cells. Nephrol Dial Transplant. 2005;20(1):50-58.

34. Gao L, et al. Restoration of E-cadherin by PPBICA protects against cisplatin-induced acute kidney injury by attenuating inflammation and programmed cell death. Lab Invest. 2018;98(7):911-923.

35. Liang J, et al. Measurement of urinary matrix metalloproteinase-7 for early diagnosis of acute kidney injury based on an ultrasensitive immunomagnetic microparticle-based time-resolved fluoroimmunoassay. Clin Chim Acta. 2019;490:55-62.

36. Zhou D, Tan RJ, Zhou L, Li Y, Liu Y. Kidney tubular $\beta$-catenin signaling controls interstitial fibroblast fate via epithelial-mesenchymal communication. Sci Rep. 2013;3:1878.

37. Tan RJ, Liu Y. Matrix metalloproteinases in kidney homeostasis and diseases. Am J Physiol Renal Physiol. 2012;302(11):F1351-F1361.

38. Grahammer F, Schell C, Huber TB. The podocyte slit diaphragm--from a thin grey line to a complex signalling hub. Nat Rev Nephrol. 2013;9(10):587-598.

39. Erkan E. Proteinuria and progression of glomerular diseases. Pediatr Nephrol. 2013;28(7):1049-1058.

40. Gorriz JL, Martinez-Castelao A. Proteinuria: detection and role in native renal disease progression. Transplant Rev (Orlando). 2012;26(1):3-13.

41. Hao S, et al. Targeted inhibition of $\beta$-catenin/CBP signaling ameliorates renal interstitial fibrosis. J Am Soc Nephrol. 2011;22(9):1642-1653.

42. Jeansson M, Björck K, Tenstad O, Haraldsson B. Adriamycin alters glomerular endothelium to induce proteinuria. $J A m$ Soc Nephrol. 2009;20(1):114-122.

43. Lee VW, Harris DC. Adriamycin nephropathy: a model of focal segmental glomerulosclerosis. Nephrology (Carlton). 2011;16(1):30-38.

44. Keppler A, et al. Plasma creatinine determination in mice and rats: an enzymatic method compares favorably with a high-performance liquid chromatography assay. Kidney Int. 2007;71(1):74-78.

45. Schmittgen TD, Livak KJ. Analyzing real-time PCR data by the comparative C(T) method. Nat Protoc. 2008;3(6):1101-1108.

46. Rush BM, Small SA, Stolz DB, Tan RJ. An efficient sieving method to isolate intact glomeruli from adult rat kidney. $J$ Vis Exp. 2018;(141).

47. Vassiliadis J, Bracken C, Matthews D, O’Brien S, Schiavi S, Wawersik S. Calcium mediates glomerular filtration through calcineurin and mTORC2/Akt signaling. J Am Soc Nephrol. 2011;22(8):1453-1461.

48. Wack KE, Ross MA, Zegarra V, Sysko LR, Watkins SC, Stolz DB. Sinusoidal ultrastructure evaluated during the revascularization of regenerating rat liver. Hepatology. 2001;33(2):363-378. 